\title{
RDUS
}

Revue de DROIT UNIVERSITÉ DE SHERBROOKE

Titre : $\quad$ LE RECOURS COLLECTIF : POINT DE VUE D'UN JUGE

Auteur(s) : $\quad$ Ginette PICHÉ

Revue : $\quad$ RDUS, 1998-1999, volume 29, numéro 1-2

Pages: $\quad 67-81$

ISSN : $\quad 0317-9656$

Éditeur : $\quad$ Université de Sherbrooke. Faculté de droit.

URI : $\quad$ http://hdl.handle.net/11143/12709

DOI : https://doi.org/10.17118/11143/12709 
Page vide laissée intentionnellement. 


\section{LE RECOURS COLLECTIF : POINT DE VUE D'UN JUGE}

par Ginette PICHÉ*

SOMMAIRE

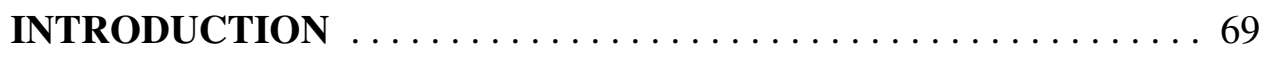

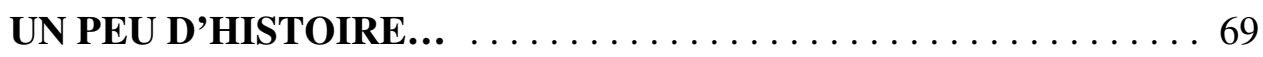

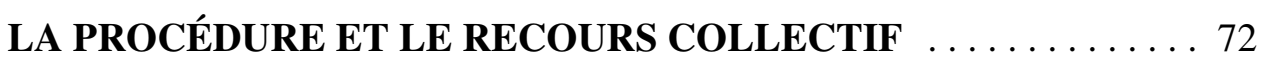

QUELQUES STATISTIQUES... . . . . . . . . . . . . . . 78

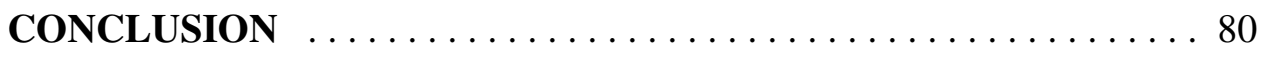

* $\quad$ Juge à la Cour supérieure du Québec. 



\section{INTRODUCTION}

Les 20 ans du recours collectif québécois. Y a-t-il de quoi fêter? C'est la question posée. L'analyse du rôle du juge saisi d'une demande de recours collectif est intéressante et permettra de répondre à la question. On verra d'abord que les juges n'ont pas énormément parlé de leur «rôle». Ils l'ont plutôt joué et de façon de plus en plus active depuis l'introduction du recours.

Dans un premier temps, je ferai un bref rappel historique de cette législation de 1978, contenue dans le Livre Neuvième du Code de procédure civile. Disons-le immédiatement, c'était, ce fut, un véritable coup de tonnerre dans l'édifice juridique. Conservatisme initial des juges, ouverture et innovations par la suite. Nous verrons, ensuite dans un deuxième temps, comment notre droit, même s'il y a souplesse, constitue toutefois un des obstacles à une plus grande ouverture de la part des juges. Ainsi, les juges ne peuvent devenir «enquêteurs» et, même s'ils demeurent conscients de la nature bien particulière de cette législation de nature sociale, ils ne peuvent aller au delà de certaines règles de procédure. Ensuite, je parlerai de statistiques. ${ }^{1}$ Finalement, je conclurai qu' on peut tirer des conclusions positives sur le rôle des juges qui, malgré les obstacles, ont su jouer un rôle actif et innovateur depuis la naissance du recours collectif. On peut donc fêter les 20 ans du recours collectif.

\section{UN PEU D'HISTOIRE...}

Il importe de rappeler d'abord que la Loi sur le recours collectif, ${ }^{2}$ entrée en vigueur le 19 janvier 1979, n'est pas née in abstracto. Elle a modifié le Code de procédure civile $e^{3}$ en venant y ajouter un livre, le Livre Neuvième. Le recours collectif participait d'une philosophie complètement opposée à ce qu'on avait connu, soit le procès entre deux individus. En 1978, on parlait même du recours collectif québécois comme du «bastion avancé de l'action de groupe

1. À ce propos, je désire remercier mon recherchiste, Me Maxime Nasr, qui a réuni pour moi ces statistiques du Fonds d'aide aux recours collectifs. Je remercie également Me Louise Ducharme pour sa collaboration.

2. L.R.Q., c. R-2.1.

3. L.R.Q., c. C-25. 
Le recours collectif:

point de vue

d'un juge

dans le monde». ${ }^{4}$ Le recours collectif allait donc permettre à une personne ou à une association de pouvoir représenter devant le tribunal, sans mandat, des personnes ayant un problème juridique semblable.

Il faut se rappeler que la Loi sur le recours collectif origine de deux lois américaines : la règle 23 des règles fédérales de procédure civile ${ }^{5}$ et les articles 901 à 909 des règles de pratique en matières civiles de l'état de New York. ${ }^{6}$ Mêmes si elles n'ont pas été complètement intégrées dans notre droit, nos législateurs s'en sont inspirés. Ainsi donc, avant l'entrée en vigueur de la Loi sur le recours collectif, le justiciable québécois, voulant agir en Cour pour d'autres personnes, n'avait qu' une unique façon de procéder, c'est-à-dire d'avoir obtenu d'elles un mandat spécifique. ${ }^{7}$

En 1978, tous les auteurs étaient, à un degré plus ou moins grand, inquiets vis-à-vis l'entrée en vigueur du recours collectif. On se questionnait beaucoup et sans être un sentiment généralisé, disons que le pessimisme régnait parfois. Il faut réaliser qu'on allait diamétralement à l'opposé de ce qu'on connaissait, de ce avec quoi on était familier. Et... vous connaissez les juges, ils sont plutôt de nature conservatrice! En fait, soyons honnêtes, c'était normal que de ressentir de l'inquiétude. Le recours collectif cadrait difficilement avec les règles ordinaires de procédure à cause de sa dimension et, surtout, des objectifs qu'il poursuivait. Notre Code de procédure civile repose en effet sur le postulat qu'un procès civil se déroule entre deux ou plusieurs personnes bien identifiées, qui soumettent elles-mêmes leurs prétentions à un juge dont le rôle essentiel est de trancher la contestation à la lumière de la preuve offerte par chacune des parties.

M. le professeur Reid écrivait, en 1984, lors de la Première Conférence Yves Pratte sur les recours collectifs, que la Loi sur les recours collectifs se fondait sur une philosophie tellement étrangère à notre droit que les

4. F. Caballero, «Plaidons par procureur! De l'archaïsme procédural à l'action de groupe» (1985) Revue trimestrielle de droit civil, 247.

5. $\quad$ Federal Rules of Civil Procedure, r. 23, 39 F.R.D. 98 (1966).

6. N.Y. Civ. Pract. Law, art. 901-909 (N.Y. Sess. Law 1975).

7. $\quad$ Art. 59 ou 67 Code de procédure civile. 
codificateurs lors de la rédaction de notre Code de procédure avaient même, en 1964, rejeté d'emblée l'hypothèse d'y inclure le recours collectif. ${ }^{8}$

Enfin, je trouve intéressant de rappeler que c'est un tout nouveau gouvernement, se disant social-démocrate, qui est à l'origine de cette loi. On voulait, comme le rappelle $\mathrm{M}$. le professeur Reid en citant les commentaires du ministre :

«[...] donner aux citoyens un instrument, un recours, un moyen de procédure qui leur permette d'obtenir justice et aussi d'obtenir individuellement et collectivement le remboursement des sommes illégalement arrachées.»9

On voulait introduire «comme une espèce de notion de sagesse» dans notre droit. On parlait donc de «réforme sociale» au delà de réforme du droit tout court.

Ce bref rappel historique est à mon avis important pour comprendre et voir comment le rôle du juge a été perçu et a évolué au cours des 20 dernières années. En fait, le recours collectif se voulait plus qu'un simple véhicule procédural pour les consommateurs leur permettant de faire valoir en justice certains droits. Dans son volume sur Le recours collectif comme voie d'accès à la justice pour les consommateurs, ${ }^{10} \mathrm{M}$. le professeur Lafond explique que le recours collectif atteint l'essence des droits des consommateurs «en participant à la réalisation des objectifs du droit matériel». Car, dira-t-il, «sans cette procédure, leur mise en œuvre risque de demeurer à l'état théorique». Pour le professeur Lafond, «elle vient bouleverser plusieurs principes liés au droit substantiel, en plus de remettre en question le rôle traditionnel du juge.» ${ }^{11}$ Il rappelle que l'auteur Abram Chayes disait que «la fonction judiciaire puise sa légitimité dans la réponse qu'elle apporte au profond besoin de justice dans

8. H. Reid, «Les conditions d'autorisation du recours collectif québécois» dans Les recours collectifs en Ontario et au Québec, Actes de la première conférence Yves Pratte (1991), Montréal, Wilson \& Lafleur Ltée, 1992.

9. J.E. Commission permanente de la justice, vol. 20, n 34, le 16 mai 1978, p. 1474.

10. P.-C. Lafond, Le recours collectif comme voie d'accès à la justice pour les consommateurs, Montréal, Les Éditions Thémis, 1996.

11. Ibid. à la p. 13 . 
notre société ${ }^{12} \gg$. M. le professeur Lafond ajoute que l'échec ou le succès de la formule québécoise du recours collectif réussit, jusqu'à un certain point, à mettre cette légitimité à l'épreuve.» ${ }^{13}$

\section{LA PROCÉDURE ET LE RECOURS COLLECTIF}

Sur ce point, s'il est exact d'affirmer que le rôle traditionnel du juge a été bouleversé, il demeure que celui-ci doit suivre la procédure mise en place par le législateur. Il ne peut l'outrepasser sous prétexte que l'on parle de justice sociale et de collectivisation du recours.

On doit rappeler en effet que, même dans la procédure du recours collectif, le rôle du juge n'est pas de mettre de côté les règles d'administration de la preuve pour devenir un «juge enquêteur» et ce, sous prétexte qu'il s'agit de droits collectifs et que le législateur a donné des pouvoirs étendus au juge. Le juge a un rôle bien particulier à jouer dans l'application de la Loi sur les recours collectifs. M. le professeur Denis Ferland, dans un article de la Revue du Barreau publiée en 1981, analysant plusieurs décisions, dira ceci en parlant de la requête pour autorisation :

«Les juges en particulier devront se reconnaître un rôle actif dans l'application de la loi, en général, et à cette étape des procédures en particulier, dans l'application de l'article 1005 C.p., mais sans devoir pour autant apporter à la requête pour autorisation des modifications que le requérant se refuse à apporter. [...] hormis cette réserve, le juge présidant le tribunal pourra exercer tous les pouvoirs très étendus que la loi lui confère de cette première étape des procédures.» ${ }^{14}$

Dans le jugement de Curateur public c. Syndicat national des employés de l'hôpital Saint-Ferdinand, ${ }^{15}$ M. le juge Lesage expliquera que :

12. A. Chayes, «The Role of the Judge in Public Law Litigation» (1976) 89 Harv. L. Rev. 1281 à la p. 1316.

13. P.-C. Lafond, supra note 10 à la p. 13.

14. D. Ferland, «Procédure civile - Droit judiciaire», (1981) 41 R. du B. 485 à la p. 494.

15. Curateur public c. Syndicat national des employés de l'hôpital Saint-Ferdinand, [1990] R.J.Q. 359 (C.S.). 
«Comme la Cour d'appel l'a énoncé dans Syndicat National des employés de l'Hôpital St-Charles Borromée c. Lapointe, la législation québécoise sur le recours collectif est de portée sociale et cherche à favoriser l'accès à la justice, particulièrement lorsqu'il s'agit de citoyens affectés dans leur état physique et mental. Cette législation est l'une des plus avancées en la matière. Le Québec est le seul État connu qui ait mis sur pied un système d'encouragement financier pour favoriser son utilisation. L'on ne peut prétendre, comme on l'a fait, que le droit fondamental à une pleine et entière défense est brimé par ce moyen exceptionnel. [...]

[...] Le Tribunal est invité à procéder par regroupements et s'y refuser constituerait un déni de justice. Qu'on soit d'accord ou non, le législateur a voulu faire jouer au tribunal un rôle nouveau dans l'exercice d'une justice collective. Ce rôle se traduit par le contrôle du recours, le contrôle de la procédure, la discrétion dans les solutions et les formes d'exécution, le procès par étapes et, surtout, la globalisation du litige par le traitement collectif, en tout ou en partie, des rapports individuels.» ${ }^{16}$ [Je souligne]

Dans un article fort intéressant publié dans la Revue du Barreau en 1986, Me Louise Ducharme, du Fonds d'aide aux recours collectifs, traite du rôle du tribunal en matière de recours collectifs. Elle cite un précédent important concernant la procédure et le rôle créateur incombant au juge. Le tribunal, dira-t-elle :

«... peut être appelé à jouer un rôle créateur pour faciliter le déroulement et la preuve d'un recours compte tenu des circonstances particulières de chaque dossier.» ${ }^{17}$

Selon elle, le jugement Comartin $^{18}$ prononcé par M. le juge Greenberg, marque une étape très importante dans l'évolution du recours collectif québécois :

16. Ibid. à la p. 370 .

17. L. Ducharme, «Le rôle du tribunal en matière de recours collectif : un précédent important», (1986) 46 R. du B. 824 à la p. 826.

18. Comartin c. Bordet (Euro-American Voyages), [1984] C.S. 584. 
«Le jugement a en effet le mérite d'énoncer très clairement les considérations qui doivent guider le tribunal lorsqu'il est appelé à exercer sa discrétion judiciaire [...].

[...) le jugement Comartin démontre qu'il est possible d'adapter les règles habituelles de preuve et de procédure pour tenir compte de la nature du recours collectif et des faits particuliers d'un dossier et ce, dans le respect des droits des parties et sans les complications administratives que l'on est très souvent tenté d'accoler au recours collectif.» ${ }^{19}$

La Cour d'appel, dans l'arrêt Tremaine, ${ }^{20}$ énoncera que le législateur «n'a pas voulu limiter le recours collectif à des stéréotypes» et que «la frontière est souvent fragile entre le droit substantiel et le droit judiciaire, le second étant au service du premier». ${ }^{21}$

Dès 1980 d'ailleurs, la Cour d'appel, dans l'arrêt de Syndicat national des employés de l'Hôpital St-Charles Borromée c. Lapointe, ${ }^{22}$ avait édicté que :

«La loi sur le recours collectif, [...] est évidemment une loi à portée sociale : elle favorise l'accès à la justice à des citoyens qui ont des problèmes communs dont la valeur pécuniaire peut souvent être d'une modicité relative et qui, en raison des circonstances particulières de leur état individuel, n'oseraient pas ou ne pourraient pas de façon appropriée mettre en marche le processus judiciaire.» ${ }^{23}$ [Je souligne]

L'examen des décisions de nos tribunaux montre qu'à partir de 1990, les juges parleront de plus en plus du rôle du tribunal en matière de recours collectif. Ainsi, en 1992, M. le juge Trotier dans la cause de Delaunais c. Québec (P.G.), ${ }^{24}$ expliquera en parlant de l'art. 1025 C.p.c. :

19. L. Ducharme, supra note 17 à la p. 832.

20. Tremaine c. A.H. Robins Canada Inc., (le 3 octobre 1990) Québec 200-09-000208-873, J.E. 90-1642 (C.A.) [ci-après Tremaine].

21. J.-C. Lafond, supra note 13 à la p. 343.

22. Syndicat national des employés de l'Hôpital St-Charles Borromée c. Lapointe, [1980] C.A. 568.

23. Ibid.

24. [1992] R.J.Q. 1578. 
«[...] nous avons rappelé le rôle agressif du Tribunal en la matière : il doit s'assurer que toute transaction sous l'empire de 1025 C.P. sert réellement l'intérêt des membres absents ; ici, ils se seraient battus à toutes fins pratiques pour un symbole. ${ }^{25}$

Dans la cause de Clavel c. Productions musicales Donald K. Donald Inc., ${ }^{26} \mathrm{M}$. le juge Hébert examine le contenu d'un règlement intervenu à quelques jours d'un procès qui devait durer 17. M. le juge Hébert donnera son accord au règlement après un examen et une analyse fouillés de la distribution proposée du reliquat de $300000 \$$.

Mme la juge Rousseau-Houle, dans l'arrêt Nadon c. Ville d'Anjou, ${ }^{27}$ rappellera encore que :

«[...] le recours collectif a une portée sociale et vise à fournir l'accès à la justice à des citoyens qui ont des problèmes communs dont la valeur pécuniaire peut souvent être d'une modicité relative et qui n'oseraient ou ne pourraient pas de façon appropriée mettre en marche le processus judiciaire. ${ }^{28}$

En 1995, la Cour d'appel statuera qu'il ne faut pas par ailleurs :

«[...] sacrifier la représentation adéquate à l'élitisme afin de favoriser l'exercice du recours collectif. D'ailleurs, les affaires Guilbert c. Vacances sans Frontière ltée,$^{29}$ Château c. Placements Germarich inc. ${ }^{30}$ et Lasalle c. Kaplan ${ }^{31}$ illustrent cette tendance de notre cour à privilégier une approche libérale dans le choix du représentant.» ${ }^{32}$

25. Ibid. à la p. 1581 .

26. (le 19 janvier 1996) Montréal 500-06-000010-922, J.E. $96-582$ (C.S.).

27. [1994] R.J.Q. 1823.

28. Ibid. aux pp. 1827-1828.

29. [1991] R.D.J. 513 (C.A.).

30. [1990] R.D.J. 625 (C.A.).

31. [1988] R.D.J. 112 (C.A.).

32. Greene c. Vacances Air Transat inc., [1995] R.J.Q. 2335 à la p. 2338. 
Le recours collectif:

point de vue

d'un juge

Toutefois, malgré ces arrêts parlant de «loi à portée sociale», en 1997, la Cour supérieure dira encore, dans la cause de Hamer c. La Reine, ${ }^{33}$ que le recours collectif est d'abord et avant tout un «moyen de procédure» permettant à un membre d'agir en demande sans mandat pour le compte de tous les membres. Dans ce jugement, M. le juge Lebel reprend alors un article publié dans les Cahiers de droit en 1980 :

«Le recours collectif n'est pas un droit (jus); c'est un moyen. [...] Ce n'est qu'un mécanisme particulier qui vient s'appliquer, pour la "collectiviser", à une façon déjà existante d'exercer un droit déjà existant. C'est un moyen de procédure de deuxième niveau, comme le sont la jonction des demandes, de parties ou d'action.» ${ }^{34}$

Ainsi, malgré que les juges parlent souvent du rôle actif qui est le leur, de leur rôle «agressif», de «loi à portée sociale», il ne faut toutefois pas perdre de vue qu'il incombe aux parties de rechercher et de présenter leurs moyens de preuve. Dans le Précis de la preuve, Léo Ducharme rappelle que :

«Lors de l'enquête, ce sont les parties qui déterminent l'ordre de présentation de leur preuve, qui choisissent les témoins qu'elles veulent faire entendre [...]. Quant au tribunal, il se voit conférer essentiellement un pouvoir de contrôle sur le déroulement de la preuve avec, toutefois, la possibilité de prendre certaines initiatives dans l'intérêt de la justice.» ${ }^{35}$

Il appartient donc aux parties de faire valoir leurs droits (art. 2803 C.c.Q.) et ce, malgré une «insuffisance de solutions procédurales classiques». ${ }^{36}$ La Cour d'appel rappellera d'ailleurs à ce sujet, dans l'arrêt récent (24 août 1998) de Technologie Labtronix Inc. c. Technologie Micro Contrôle Inc. ${ }^{37}$ que même si le rôle traditionnel du juge a grandement évolué et qu'il a une plus grande latitude dans l'administration de la preuve, (il peut même jouer le rôle

33. (le 12 mai 1997) Québec 200-06-000001-969, J.E. 97-1236 (C.S.).

34. M. Bouchard, «L'autorisation d'exercer le recours collectif», (1980) 21 Cahier de droit 855 aux pp. 864 et s.

35. L. Ducharme, Précis de la preuve, $5^{\mathrm{e}}$ éd., Montréal, Wilson \& Lafleur, 1996 à la p. 46.

$36 . \quad$ J.-C. Lafond, supra note 13 à la p. 55.

37. (le 24 août 1998) Montréal 500-09-004365-979 (C.A.) [ci-après Labtronix]. 
de «remédiateur» ${ }^{38}$ et signaler une lacune dans la preuve et la procédure), il demeure que le juge ne peut s'immiscer dans le débat et «conduire» lui-même la preuve. La Cour d'appel précisera même que :

«N'est pas [...] confié au juge le mandat d'un commissaire-enquêteur investi de pouvoirs qui ne participent pas du régime accusatoire et contradictoire, comme le droit d'assigner des témoins et de procéder à des saisies.» ${ }^{39}$

Analysant l'article 2810 C.c.Q., la Cour d'appel énoncera que l'on peut considérer que :

«[...] l'article 2810 C.c.Q. vient parfaitement s'intégrer dans un système qui, ultimement, confie au juge une mission dynamique et progressiste sans toutefois brimer "le lien juridique d'instance".» ${ }^{40}[\mathrm{Je}$ souligne]

Donc, même si les dispositions relatives au Livre Neuvième ont été interprétées de façon généreuse, il reste que le juge œuvrant dans un système contradictoire ne peut chambarder toutes les règles. La procédure du recours collectif est venue bouleverser le droit, la procédure et la justice ; les juges l'ont compris. Ils ne peuvent cependant travailler que dans le cadre procédural en vigueur, tout en prenant en considération la portée de la loi. C'est d'ailleurs ce qu'ils ont fait dans la plupart des cas. M. le professeur Hubert Reid rappelle ceci au sujet des règles de procédure :

«À cause de leur formalisme actuel, nos règles de procédure accroissent inévitablement le coût des procès, compliquent l'administration de la justice par la multiplication du nombre d'instances et donnent ouverture à la possibilité de jugements contradictoires sur des questions similaires. De plus, lorsque le litige impose aux parties l'obligation de soumettre des preuves techniques complexes et coûteuses, le juge est souvent témoin d'un combat entre

38. P. Tessier, «La vérité et la justice», (1988) 19 R.G.D. aux pp. 29-80.

39. Labtronix, supra note 37 à la p. 37.

40. Ibid. à la p. 46. 
David et Goliath, à la différence que le Goliath de 1977 est souvent en possession de la fronde. ${ }^{41}$

Cette affirmation est exacte et plus souvent encore dans le cas des recours collectifs. Examinons quelques statistiques.

\section{QUELQUES STATISTIQUES...}

À partir de l'année 1979, jusqu'à la fin 1997, 422 dossiers de recours collectifs ont été ouverts. De ce nombre, 222 l'ont été dans les dix premières années (1979 à 1988) d'existence du recours, alors que l'on compte 200 ouvertures de dossiers au cours des dix plus récentes années (1988 à 1997) De ceci, on peut d'abord conclure que l'engouement des premières années pour cette nouvelle voie procédurale ne s'est pas démenti avec le temps et qu'aujourd'hui encore cette avenue reçoit toutes les considérations auxquelles elle a droit.

De ces 422 dossiers ouverts, 106 ont donné lieu à un jugement autorisant l'exercice du recours collectif, tandis que dans 109 cas, cette autorisation était refusée. Il est intéressant d'observer que d'une décennie à l'autre, on constate un sérieux changement de cap. En effet, alors qu'au cours des années 1979 à 1988 le nombre de requêtes pour autorisation d'exercer un recours collectif auxquelles les tribunaux faisaient droit (45) était inférieur au nombre de requêtes rejetées (62), la deuxième décennie est marquée par la tendance inverse. Durant cette période, 72 autorisations ont été données, alors que les requérants ne devaient essuyer que 56 refus.

Cette nouvelle tendance peut s'expliquer par plusieurs théories. Je me permets toutefois de poser l'hypothèse que c'est toute la philosophie actuelle des tribunaux face aux recours collectifs qui est responsable de ce revirement. À cet effet, il est éloquent de mettre en relation deux obiter de notre Cour d'appel. Un

41. H. Reid, «Le recours collectif - La mise en œuvre judiciaire du recours collectif : questions et hypothèses», Barreau du Québec, Formation permanente (1978-1979), Cours \#38 à la p. 128. 
premier donné en 1984 et un deuxième, 10 ans plus tard. Regardons-les. Sous la plume du juge Mayrand, en 1984, la Cour d'appel disait :

«[...] Se contenter d'une vague possibilité que ces faits essentiels existent, plutôt que d'une apparence sérieuse de leur existence, inviterait les justiciables à abuser de la procédure exceptionnelle du recours collectif.» ${ }^{42}$ [Je souligne]

En 1994, le juge Nichols, s'inspirant des propos du juge en chef Bisson dans l'affaire Tremaine, ${ }^{43}$ marquait définitivement une nouvelle direction :

«Le recours collectif est un véhicule procédural comme il y en a plusieurs autres dans le Code et il est disponible lorsque les conditions d'exercice se rencontrent. C'est donc à tort que les appelants traitent le présent recours de "recours exceptionnel"..»" [Je souligne]

Le recours collectif n'est définitivement plus un «recours exceptionnel». Il fait maintenant partie de nos mœurs.

Voyons maintenant le sort réservé aux actions procédant au fond : 35 jugements rendus quant au fond en matière de recours collectif durant ces dernières dix-neuf années, seulement 9 ont été rejetées. Si on ajoute à cela les règlements hors cour, tant au stade de la requête en autorisation que de la cause au mérite, ainsi que les désistements, qui pour une bonne part d'entre eux constituent des succès pour les membres, ${ }^{45}$ on est en droit de conclure que, du point de vue du justiciable, l'avènement du recours collectif était une nécessité et a donné des résultats encourageants.

42. Vignola c. Chrysler Canada Ltée, [1984] R.D.J. 327 à la p. 330 (C.A.).

43. Tremaine, supra note 20.

44. Syndicat national des employés de l'Hôpital St-Ferdinand c. Québec (Curateur public), [1994] R.J.Q. 2761 à la p. 2781 (C.A.).

45. Le Fonds d'aide aux recours collectifs, Rapport annuel 1996-1997, Montréal, Fonds d'aide au recours collectif, 1997 à la p. 16 affirme : «Il faut encore tenir compte, pour cerner le taux de succès ou d'échec du recours, au niveau de l'action comme telle, [que] les dossiers réglés [hors cour] et une bonne part de ceux pour lesquels il y a eu désistement constituent des succès pour les membres.» 
Le recours collectif:

point de vue

d'un juge

Finalement, il est frappant de constater que le premier règlement hors cour n'est survenu qu'en 1989, soit plus de dix ans après l'entrée en vigueur de la Loi. Depuis ce temps, les règlements s'accumulent et constituent aujourd'hui le principal mode de finalisation de ce type de litige. Depuis huit ans le nombre de règlement hors cour est supérieur au nombre de jugements finals en cette matière. Doit-on s'en inquiéter? Je crois que non et qu'au contraire, il faut s'en réjouir. C'est d'ailleurs en accord avec la tendance actuelle qui voit les individus essayer de régler hors cour leur litige plutôt que de le faire traîner devant les tribunaux.

\section{CONCLUSION}

Avec la Loi sur le recours collectif, le législateur a fait cadeau d'un rôle très exigeant au juge. Un rôle qui a d'ailleurs évolué et où le juge de première instance a peut-être été un peu trop prudent au début. Ce seront la Cour d'appel et la Cour suprême qui amèneront véritablement une interprétation large de la nouvelle législation. Le juge de première instance est ainsi devenu plus créatif avec les années et son ouverture a encouragé beaucoup de règlements. Je vous soumets cette hypothèse : les juges québécois ont, malgré les embûches, réussi à être non seulement ouverts, mais même dans de nombreux cas, innovateurs et éveillés à l'esprit de cette procédure qu'est le recours collectif. Des progrès peuvent et doivent être faits encore, mais la voie est tracée. Les citoyens ne doivent pas avoir peur de s'y intéresser, d'en être partie afin que le recours collectif obtienne véritablement tout le succès qu'il mérite. Il faut se rappeler par ailleurs que le juge n'est pas le législateur et qu'il a dû - et doit encore composer avec de nombreux obstacles. Le juge deviendra-t-il de plus en plus un conciliateur? Devrait-il l'être et jouer ce rôle?

On sait par ailleurs que les règles de procédure sont destinées à faire apparaître le droit (art. 2 C.p.c.) et à en assurer la sanction. La Cour d'appel l'a rappelé récemment, tout en notant que le juge n'a pas pour rôle d'être enquêteur. À mon avis, ceci ne peut et ne veut pas dire que le juge n'a pas un rôle où interviennent éthique et justice sociale. Le juge doit être conscient de cela et demeurer ouvert et innovateur. Il y a ici un instrument visant à servir la justice sociale et c'est ce que le juge, dans sa fonction la plus noble et la plus belle doit réaliser : rendre la justice avec courage et trancher en faveur de la «cause juste» 
quels que soient les enjeux. Les vingt ans du recours collectif québécois ont permis à notre société de régler certains conflits ayant des enjeux réels, mais il y a encore beaucoup à faire. Les avocats et les juges ont été des acteurs importants du recours collectif et même si plusieurs améliorations doivent venir, il y a lieu d'être fiers de ce qui a été accompli jusqu'ici. Il y a lieu de fêter les vingt ans du recours!

En terminant, j'aimerais vous laisser avec quelques mots du philosophe et chef d'État de la République Tchèque, Václav Havel qui, dans une conférence prononcée à Hiroshima en décembre 1995, disait :

«... si l'humanité peut espérer un avenir favorable, cet espoir repose surtout dans l'éveil d'une responsabilité générale dont les racines s'ancrent infiniment plus profond que dans le monde des intérêts terrestres transitoires et temporaires.» ${ }^{46}$

C'est ce que je nous souhaite à tous. 